\title{
Patient Specification Quality Assurance for Glioblastoma Multiiorme Brain Tumors Treated with Intensity Modulated Radiation Therapy
}

\author{
H. I. Al-Mohammed ${ }^{凶}$ \\ King Faisal Specialist Hospital \& Research Centre, Dept. of Biomedical Physics, Riyadh 11211, Saudi Arabia
}

$\triangle$ Corresponding author: Dr. Huda I. Al-Mohammed, King Faisal Specialist Hospital \& Research Centre, Dept. of Biomedical Physics, MBC \# 03, POB 3354, Riyadh 11211, Saudi Arabia. Email: hmohamed@kfshrc.edu.sa; Tel: +966(1) 464-7272, Ext 35052

(c) Ivyspring International Publisher. This is an open-access article distributed under the terms of the Creative Commons License (http://creativecommons.org/ licenses/by-nc-nd/3.0/). Reproduction is permitted for personal, noncommercial use, provided that the article is in whole, unmodified, and properly cited.

Received: 2010.12.27; Accepted: 2011.06.02; Published: 2011.08.02

\begin{abstract}
The aim of this study was to evaluate the significance of performing patient specification quality assurance for patients diagnosed with glioblastoma multiforme treated with intensity modulated radiation therapy. The study evaluated ten intensity modulated radiation therapy treatment plans using $10 \mathrm{MV}$ beams, a total dose of $60 \mathrm{~Gy}$ (2 Gy/fraction, five fractions a week for a total of six weeks treatment). For the quality assurance protocol we used a two-dimensional ionization-chamber array (2D-ARRAY). The results showed a very good agreement between the measured dose and the pretreatment planned dose. All the plans passed $>95 \%$ gamma criterion with pixels within $5 \%$ dose difference and $3 \mathrm{~mm}$ distance to agreement. We concluded that using the 2D-ARRAY ion chamber for intensity modulated radiation therapy is an important step for intensity modulated radiation therapy treatment plans, and this study has shown that our treatment planning for intensity modulated radiation therapy is accurately done.
\end{abstract}

Key words: Photon-beam dose calculation; quality assurance, intensity modulated radiation therapy, dose verification, gamma index, glioblastoma multiforme.

\section{Introduction}

Glioblastoma multiforme (GBM) is the most common malignant tumor of the subcortical white matter of the cerebral hemisphere in adults. It accounts for $12 \%-15 \%$ of all primary brain tumors [1]. The treatment of GBM involves surgical resection, which is the first therapeutic modality for GBM, followed by radiotherapy that may be accompanied by adjuvant chemotherapy [2]. In general, patients with GBM have poor prognosis with about $20 \%$ of patients surviving beyond 2 years [2]. However, some factors may be associated with a longer survival rate. These factors include younger age, gender, unilateral tumor, a high Karnofsky score, size of the tumor, extent of disease, and adjuvant treatments with chemotherapy such as temozolomide (TMZ) [3].
In recent years, the development of state-of-the-art radiation therapy and recent advances in chemotherapy have increased the chances for a good prognosis for GBM patients [4]. Intensity modulated radiotherapy (IMRT) allows for a high dose of radiation to be delivered to the tumor while permitting maximal sparing of normal tissue which reduces the radiation toxicity [5-9]. In the case of glioblastoma multiforme, IMRT has shown the potential to deliver a highly conformal dose to the target while minimizing dose to the organs at risk (OAR) such as the optic chiasm [10]. This can allow for dose escalation, while on the other hand, also increase local control $[6,7,11]$. Treatment with IMRT fields involves the complex movement of a multileaf collimator (MLC) which 
consists of many small and irregular multileaf fields or segments that can be delivered in two main modalities, namely segmental IMRT step-and-shoot (SS) or dynamic IMRT (sliding window) [12]. In the IMRT step-and-shoot (SS) technique, the shape of the leaves stays constant while the radiation beam is on and changes when the radiation beam is off, while in the dynamic sliding window technique each leaf pair moves continuously in one direction with independent speeds while the radiation beam is on [13].

IMRT dose distributions have the characteristics of complex 3-dimensional dose gradients and a timedependent fluence delivery [14]. These complex characterizations make quality assurance for every IMRT treatment compulsory. The goals of the pretreatment quality assurance are to assure the precision of the IMRT treatment plan and the application of the prescribed dose from the plan [13]. As a consequence of the complexity of the IMRT technique, additional dose checking methods are required to confirm the exact calculation of the dose for all patients treated with IMRT $[15,16]$. The most common applied dose evaluation tools encompass a direct comparison of dose differences that have a comparison of distance-to-agreement (DTA) between the measured dose and the calculated dose distributions from the planning system $[16,17]$.

The checking procedure for IMRT includes several steps which then lead to the quality assurance (QA) for the whole IMRT treatment plan. These steps include the multileaf collimator (MLC) QA, the measurements of individual patient fluence maps, the calibration of the tools used, and the reproducibility of patient positioning [18]. The planned dose fluence is compared with deliverable dose fluence, usually by using a two-dimensional array with ionization chambers, electronic portal imaging devices (EPID), or radiochromic film named "Gafchromic EBT film" $[19,20]$. In this study we used a two-dimensional array with 729 ionization chambers, which is a portal dose device for IMRT plan verification.

\section{Materials and Methods}

Our IMRT pretreatment dose verification method consisted of the following two independent measurements: first, point dose measurements at the isocenter using a two-dimensional detector matrix with 729 ionization chambers (2D-ARRAY) (PTW, Freiburg, Germany); and second, using RadCalc (RadCalc, Lifeline Software, Inc., Tyler, TX) to check independent monitor units (MU) for each beam. Pretreatment IMRT plans for ten patients diagnosed with GBM brain tumors were selected. For each of the ten pretreatment plans, verification IMRT plans were created using a Varian Eclipse external beam treatment planning system (Eclipse TPS) (8.1.18, Varian Medical Systems Inc., Palo Alto, CA). All IMRT verification plans have the same dosimetric parameters of the original plans. The dose was calculated using the Pencil Beam Convolution (PBC) algorithm built-in in the 3-dimensional treatment planning system. The verification plan for each patient was created to start the verification process. All treatment parameters, i.e., monitor units, field sizes, gantry angles, and leaf motion instructions, are stored in the database of ARIA Oncology (Varian Medical Systems Inc., Palo Alto, CA), which is an oncology-specific electronic medical record (EMR) that manages clinical activities such as radiation treatment.

The system is connected through a network to all of the treatment units. The two-dimensional array used in this investigation (2D-ARRAY) is equipped with 729 vented plane parallel ion chambers. Each detector covers an area of $5 \times 5 \mathrm{~mm}^{2}$ and the measuring depth is at $5 \mathrm{~mm}$ water. The sensitive volume of each chamber is $0.125 \mathrm{~cm}^{3}$. These ionization chambers are uniformly arranged in a $27 \times 27$ matrix with an active area of $27 \times 27 \mathrm{~cm}^{2}$ and dimensional area of 22 $\mathrm{mm} \times 300 \mathrm{~mm} \times 420 \mathrm{~mm}$, interface: $80 \mathrm{~mm} \times 250 \mathrm{~mm} \times$ $300 \mathrm{~mm}$, allowing absolute dose and dose rate measurements of high-energy photon beams.

The 2D-ARRAY chamber is calibrated using a setup of $10 \mathrm{~cm} \times 10 \mathrm{~cm}$ field size, $100 \mathrm{MU}, 10 \mathrm{MV}$ beams at a depth of $10 \mathrm{~cm}$, and a dose rate of 300 cGy/MU. In favor of the verification plans, the 2D-ARRAY setup consists of three solid water slabs of polymethyl methacrylate (PMMA) with deferent thicknesses of $3 \mathrm{~cm}, 4 \mathrm{~cm}$ and $1 \mathrm{~cm}$.

The $3 \mathrm{~cm}$ thickness slab was used as a backscatter phantom, where the other two slabs with a total thickness of $10 \mathrm{~cm}$ was used as a buildup phantom. The 2D-ARRAYchamber center was aligned with the isocenter of the plan. The 2D planar dose distribution was calculated at a $10 \mathrm{~cm}$ depth in the phantom using $1 \mathrm{~mm}$ pixel-dose grid resolution, and the point dose was calculated at the isocenter; whereas the reference point was $5 \mathrm{~mm}$ behind surface. The individual fields are radiated in gantry and collimator position of $0^{\circ}$ on the array and source-to-surface distance (SSD) of 94.5 $\mathrm{cm}$, using dynamic multileaf collimation on a Varian linear accelerator Clinac 2100EX equipped with the 120-leaf Millennium MLC (Varian Medical Systems Inc., Palo Alto, CA). The MLC system has 60 pairs of leaves in each bank and MLC leaf width projected at isocenter is $1 \mathrm{~cm}$. The leaf ends are rounded. The 2D-ARRAY chamber is connected to a laptop outside the treatment room which runs software from PTW. 
The software is MatrixScan (PTW-Verisoft 3.1) which records the measurements with the 2D-ARRAY. Prior to the treatment the temperature, pressure, and a correction factor for the machine is entered into the MatrixScan software. Each beam of the treatment plan is delivered to the 2D-ARRAY chamber, thus the dose at some reference points can be calculated. The measured dose distributions were then compared to those calculated by the Eclipse TPS. The IMRT treatment plans for each of the ten patients consisted of 5 to 11 beams using $10 \mathrm{MV}$ beams with total dose of $60 \mathrm{~Gy}$ and a dose of $2.0 \mathrm{~Gy}$. Every field is irradiated in each plan one after another on the 2D-ARRAY without interruptions or entering the treatment room and the combined dose is measured, reflecting the contribution from all beams for every plan. The measured dose by 2D-ARRAY was compared with the planned dose using verification software based on the gamma index criterion $[19,20]$. Comparisons between measured and calculated dose distributions are reported as dose difference (DD) (pixels within 5\%), distance to agreement (DTA) (3 $\mathrm{mm})$, as well as gamma values $(\gamma)$ (dose $3 \%$, distance 3 $\mathrm{mm})$.

\section{Statistical analysis}

Data from each sample were run in duplicate and expressed as means $\pm \mathrm{SD}$ (cGy, $\mathrm{n}=10$ patients). Means were considered significantly different if $P<$ 0.05 . Statistical analysis was performed by means of a GraphPad Prism ${ }^{\mathrm{TM}}$ package for personal computers
(GraphPad Software, Inc., San Diego, USA) and figures were drawn using the GraFitTM package for personal computers (Erithacus Software Limited, Surrey, UK). An ANOVA analysis using Tukey's test for multiple comparison tests was performed on the data.

\section{Results}

In this study we evaluated our QA system for IMRT plans that are going to be used to treat patients with GBM brain tumors. Presently, we perform routine QA measurements for each IMRT patient either immediately prior to the treatment or shortly after the first treatment. Table 1 shows the total number of IMRT fields for the ten selected treatment plans measured, the fractional dose for each plan, and the fractional measured dose by 2D-ARRAY. Table 1 also shows the percentage dose different between the TPS and the VeriSoft software measured dose in addition to the percentage of pixels passing gamma criterion. The overall study result is shown in Figure 1. The average dose difference between planned and measured dose was $-0.28 \%$ with a standard deviation of 1.06. Considering that the passing criteria for IMRT plans is based on the percentage of pixels passing gamma index $>95 \%$ within dose difference (pixels is within $5 \%$ ), and distance to agreement dose is $3 \mathrm{~mm}$, all of our ten selected treatment plans passed the gamma analysis test with an average of $97 \%$ pixels with an SD of 0.015.

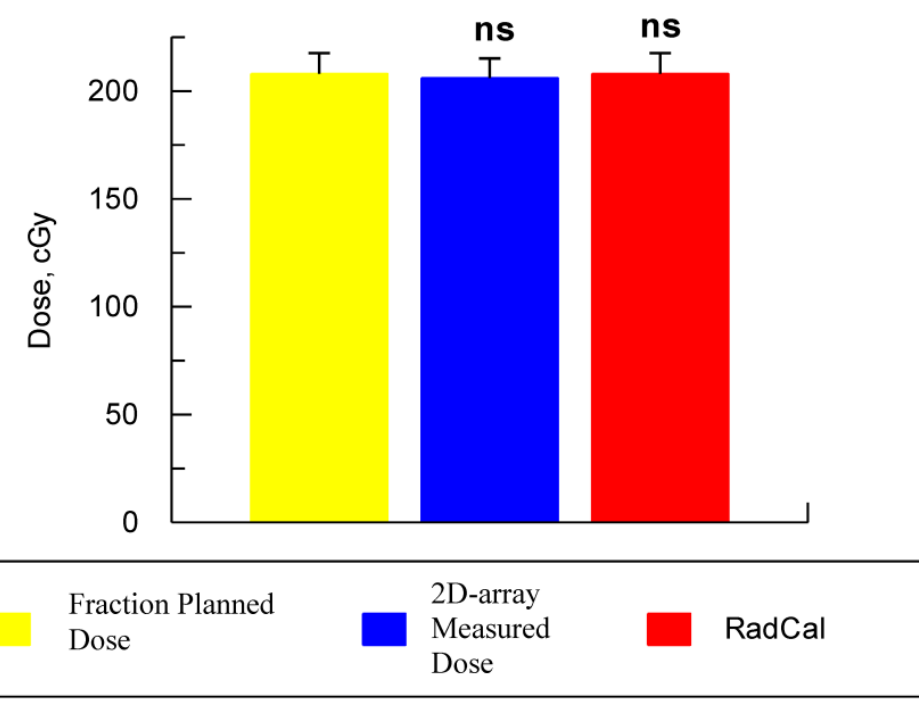

Figure 1: This graph shows the mean \pm SD for the 10 patients of the prescribed dose and measured doses using the 2D-ARRAY ion chamber. There was no significant difference (ns) between the target fraction planned dose using TPS with either 2D-ARRAY or the dose that been calculated using RadCal. (ANOVA analysis, Tukey's test for multiple comparison tests). 
Table 1: This data shows the fractional dose for the planned and measured radiation treatment, the RadCalc calculations, the \% dose difference between TPS and VeriSoft software measured dose, and the \% of pixels passing gamma criterion for the 10 patient treatment plans

\begin{tabular}{|c|c|c|c|c|}
\hline Patient's fields numbers & $\begin{array}{l}\text { Fraction Planned } \\
\text { Dose, cGy }\end{array}$ & $\begin{array}{l}\text { 2D-ARRAY } \\
\text { Measured dose, } \\
\text { cGy }\end{array}$ & $\begin{array}{l}\% \text { dose difference between } \\
\text { TPS and VeriSoft software } \\
\text { measured dose }\end{array}$ & $\begin{array}{l}\% \text { of pixels passing gamma } \\
\text { criterion }\end{array}$ \\
\hline 5 & 200.0 & 199.80 & $-0.10 \%$ & $99 \%$ \\
\hline 8 & 219.2 & 219.85 & $0.29 \%$ & $97 \%$ \\
\hline 11 & 219.2 & 218.90 & $-0.27 \%$ & $96 \%$ \\
\hline 9 & 200.0 & 200.70 & $0.35 \%$ & $97 \%$ \\
\hline 8 & 219.2 & 218.50 & $-0.31 \%$ & $97 \%$ \\
\hline 11 & 200.0 & 200.00 & $0.00 \%$ & $100 \%$ \\
\hline 11 & 200.0 & 199.80 & $-0.10 \%$ & $99 \%$ \\
\hline 8 & 200.0 & 198.90 & $-0.55 \%$ & $97 \%$ \\
\hline 5 & 200.0 & 199.20 & $-0.40 \%$ & $97 \%$ \\
\hline 7 & 200.0 & 200.50 & $0.25 \%$ & $95 \%$ \\
\hline IMRT fields total of $=83$ & $\begin{array}{l}\text { Average Dose }=205.73 \\
\text { cGy }\end{array}$ & $\begin{array}{l}\text { Average Dose }=205.615 \\
\text { cGy }\end{array}$ & $\mathrm{SD}=0.00307$ & $\mathrm{SD}=0.0151$ \\
\hline
\end{tabular}

\section{Discussion}

Glioblastoma multiforme (GBM) is the most frequently encountered and most malignant form of brain tumor, with a poor prognosis and low life expectancy [21] Intensity modulated radiation therapy (IMRT) is a new development of conformal radiotherapy which shows a better outcome for treatment, with a better sparing of the normal brain tissue and other critical structures [19]. IMRT treatment plans are complex radiotherapy treatment plans that require a comprehensive QA field-by-field in addition to complex analysis methods [20, 22]. The need for the sophisticated treatment plans and measurements increases if the tumor is located in an area surrounded by healthy and critical tissues. For example, a tumor in brain is surrounded by many organs at risk (OAR) such as the brain stem and the optic chiasm [10]. In our study we evaluated our QA system of IMRT plans that we use to treat patients with GBM.

Presently, we perform routine QA measurements for each IMRT patient either immediately prior to the treatment or shortly after the first treatment, which is the protocol we use to avoid any delay for the treatment. The ten selected treatment plans were evaluated using 2D-ARRAY in addition to independent monitor unit calculations using RadCal; however, the study focused only on the measured dose by the ion chamber 2D-ARRAY. Figure 2 shows the plan dose calculated by TPS and Figure 3 shows the measured dose by the 2D-ARRAY.The results showed agreement between the measurement dose by the 2D-ARRAY and the calculated dose produced by the TPS. Figure 4 shows the overlap of the planned dose and the measured dose using the gamma index. Every point measured in these plans agreed to within $\pm 3 \%$ acceptability criteria.
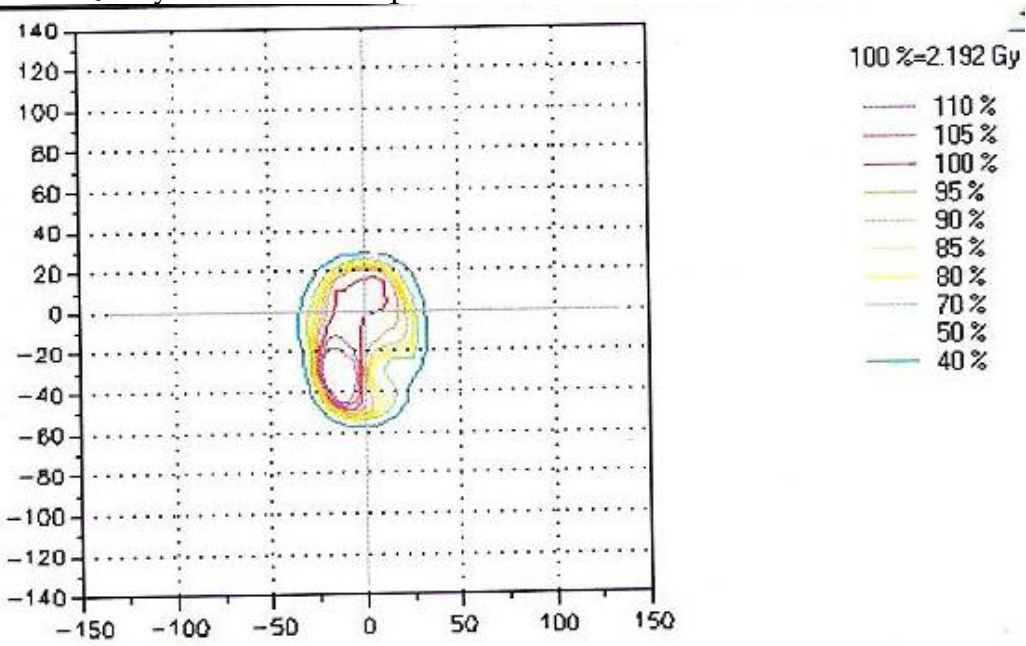

Figure 2: The chart presenting the matrix of isodose line chamber readings failing the gamma-index criterion for the planned dose by the TPS where the fractional dose is was $2.192 \mathrm{~Gy}$ 


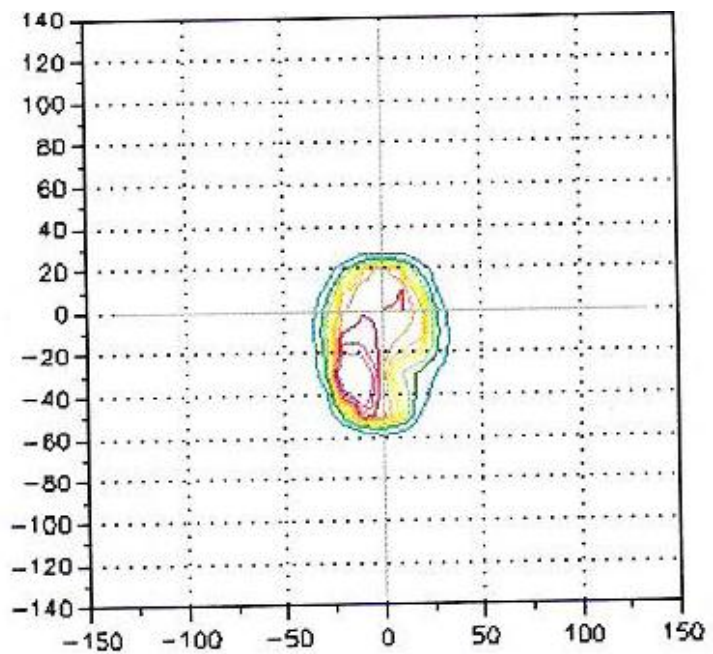

$100 \%=2.185$ Gy

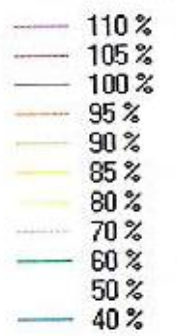

Figure 3: This chart shows the matrix of isodose lines of the measured dose by the 2D-ARRAY where the fractional dose was 2.185Gy

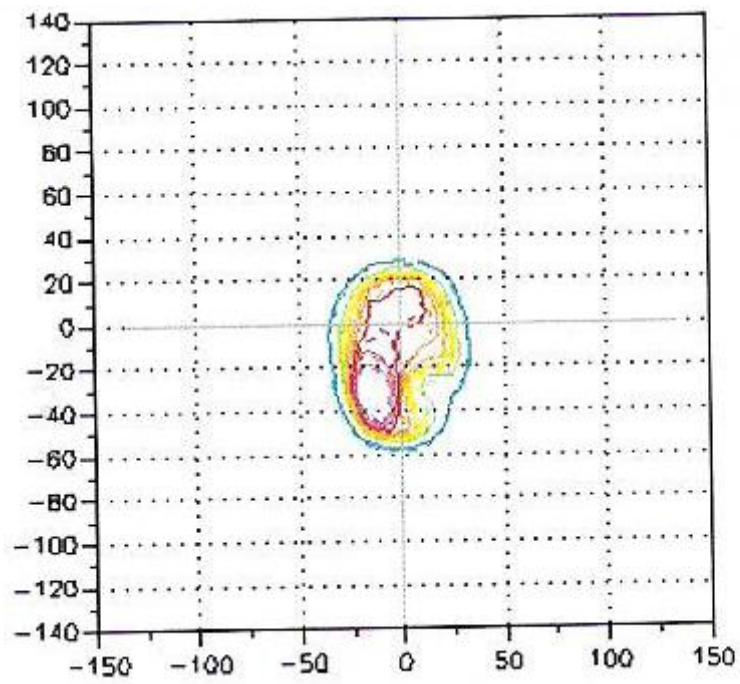

$100 \%=2.192 / 2.185$ Gy

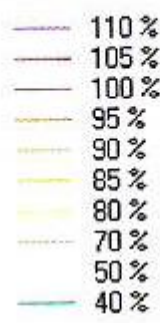

Figure 4: This chart shows the matrix of isodose compression between the planned dose in PTS and the measured dose by 2D-ARRAY ion chambers, where the matrix for the measured dose is shown in dashed lines. In this data $99 \%$ of the evaluated points passed.

All the ten selected pretreatment plans were acceptable for clinical use. The evaluation of pretreatment plans for IMRT QA is based on many factors such as patient position and patient immobilization and reproducibility; however, here we only evaluated the IMRT QA using the 2D-ARRAY ion chamber. All of our ten selected treatments plans successfully passed the gamma analysis criterion with more than $97 \%$ pixels in every defined field size for each treatment plan.

\section{Conclusion}

Patient specific dosimetric QA for IMRT plan is an important component of clinical usage of IMRT.
Our result showed a very good agreement between measurements dose and calculated dose which demonstrated that our treatment planning using IMRT is accurately done compared with the dose planned by the TPS. The 2D-ARRAY ion chamber measurement agreed with the planned dose, all the plans passed with $>95 \%$ gamma criterion with pixels under $5 \%$ dose difference and $3 \mathrm{~mm}$ distance to agreement for IMRT patient-specific quality assurance (QA). A good consistency was observed across the treatments. We concluded that using 2D-ARRAY for IMRT verification plans is a fast method and possesses all the advantages of ionization chamber dosimetry. 


\section{Acknowledgments}

The author would like to express her gratitude thanks to all of our patients who participated in this study and without whom the study cannot be completed. In addition the author would like to extend her thanks to King Faisal Specialist Hospital and Research Center, Riyadh, Saudi Arabia for their continuous support. The author would like to acknowledge the professional editing assistance of Dr. Belinda Peace.

\section{Conflict of Interest}

The authors have declared that no conflict of interest exists.

\section{References}

1. Birbilis TA, Matis GK, Eleftheriadis SG, et al. Spinal metastasis of glioblastoma multiforme: an uncommon suspect? Spine (Phila Pa 1976). 2010; 35: E264-9.

2. Fine HA. The basis for current treatment recommendations for malignant gliomas. J Neurooncol. 1994; 20: 111-20.

3. Das P, Puri T, Jha P, et al. A clinicopathological and molecular analysis of glioblastoma multiforme with long-term survival. J Clin Neurosci. 2011; 18: 66-70.

4. MacDonald SM, Ahmad S, Kachris S, et al. Intensity modulated radiation therapy versus three-dimensional conformal radiation therapy for the treatment of high grade glioma: a dosimetric comparison. J Appl Clin Med Phys. 2007; 8: 47-60.

5. Nieder C, Astner ST, Mehta MP, et al. Improvement, clinical course, and quality of life after palliative radiotherapy for recurrent glioblastoma. Am J Clin Oncol. 2008; 31: 300-5.

6. Al-Mohammed HI. The efficiency of using audio prompting method to regulate the patient's breathing during radiation therapy treatment of NSCLC. Int J Med Med Sci. 2011; 3: 1-6.

7. Al-Mohammed HI. Investigation of breathing maneuvers using free breathing and video biofeedback techniques during radiation therapy treatment for non small cell lung cancer patients. J Can Res Exp Oncol. 2010; 2: 60-71.

8. Hong TS, Ritter MA, Tome WA, et al. Intensity-modulated radiation therapy: emerging cancer treatment technology. Br J Cancer. 2005; 92: 1819-24.

9. Bhide SA, Nutting CM. Recent advances in radiotherapy. BMC Med. 2010; 8: 25.

10. Xiao WW, Huang SM, Han F, et al. Local control, survival, and late toxicities of locally advanced nasopharyngeal carcinoma treated by simultaneous modulated accelerated radiotherapy combined with cisplatin concurrent chemotherapy: long-term results of a phase 2 study. Cancer. 2011;117(9):1874-83.

11. Roeder F, Timke C, Zwicker F, et al. Intensity modulated radiotherapy (IMRT) in benign giant cell tumors--a single institution case series and a short review of the literature. Radiat Oncol. 2010; 5: 18.

12. Abate A, Pressello MC, Benassi M, et al. Comparison of IMRT planning with two-step and one-step optimization: a strategy for improving therapeutic gain and reducing the integral dose. Phys Med Biol. 2009; 54: 7183-98.

13. Saminathan S, Manickam R, Chandraraj V, et al. IMRT implementation and patient specific dose verification with film and ion chamber array detectors. Pol J Med Phys Eng. 2009; 15: 87-102.

14. Han $Y$, Shin EH, Lim C, et al. Dosimetry in an IMRT phantom designed for a remote monitoring program. Med Phys. 2008; 35 : 2519-27.
15. Soffietti R, Ruda R, Trevisan E. Brain metastases: current management and new developments. Curr Opin Oncol. 2008; 20: 676-84.

16. Oldham M, Guo P, Gluckman $G$, et al. IMRT verification using a radiochromic/optical-CT dosimetry system. J Phys. 2006; 56: 221-24.

17. Li QL, Deng XW, Chen LX, et al. The angular dependence of a 2-dimensional diode array and the feasibility of its application in verifying the composite dose distribution of intensity-modulated radiation therapy. Chin J Cancer. 2010; 29: 617-20.

18. Schneider F, Polednik M, Wolff D, et al. Optimization of the Gafchromic(TM) EBT protocol for IMRT QA. Zeitschrift für Medizinische Physik. 2009; 19: 29-37.

19. Jiang YG, Peng Y, Koussougbo KS. Necroptosis: a novel therapeutic target for glioblastoma. Med Hypotheses. 2011; 76: 350-2.

20. Boggula R, Jahnke L, Wertz $\mathrm{H}$, et al. Patient-specific 3D Pretreatment and Potential 3D Online Dose Verification of Monte Carlo-calculated IMRT Prostate Treatment Plans. Int J Radiat Oncol Biol Phys. 2010; epub.

21. Agazaryan N, Solberg TD, DeMarco JJ. Patient specific quality assurance for the delivery of intensity modulated radiotherapy. J Appl Clin Med Phys. 2003; 4: 40-50.

22. Depuydt T, Van Esch A, Huyskens DP. A quantitative evaluation of IMRT dose distributions: refinement and clinical assessment of the gamma evaluation. Radiother Oncol. 2002; 62: 309-19. 